\title{
Long-Time Behavior of Solution for a Reactor Model
}

\author{
Yantao Guo, ${ }^{1}$ Jianwei Shen, ${ }^{1}$ and Qianqian Zheng ${ }^{2}$ \\ ${ }^{1}$ Institute of Applied Mathematics, Xuchang University, Xuchang, Henan 461000, China \\ ${ }^{2}$ School of Information Science and Technology, Donghua University, Shanghai 200051, China
}

Correspondence should be addressed to Jianwei Shen; xcjwshen@gmail.com

Received 13 July 2015; Accepted 14 September 2015

Academic Editor: Remi Léandre

Copyright (C) 2015 Yantao Guo et al. This is an open access article distributed under the Creative Commons Attribution License, which permits unrestricted use, distribution, and reproduction in any medium, provided the original work is properly cited.

In this paper, we would consider the dynamical behaviors of the chemical model represented by Satnoianu et al. (2001). Using the Kuratowski measure of noncompactness method, we prove the existence of global attractor for the weak solution semiflow of system. Finally, several numerical experiments confirm the theoretical results.

\section{Introduction}

Satnoianu et al. [1] considered a chemical model which assumed that a precursor $P^{+}$is present in excess. They required further species $Q^{-}$to be present in the reactor at a concentration similar to that of $P^{+}$though this species does not take part in the reaction. Assume that $P^{+}$decays at a constant rate to form the substrate $A^{+}$via $P^{+} \rightarrow$ $A^{+}$, rate $=k_{0} p_{0}$, where $p_{0}$ is the initial concentration of the reservoir species $P^{+}$. The substrate $A^{+}$and autocatalyst $B^{+}$subsequently react by following a linear combination of quadratic and cubic steps according to the scheme

$$
\begin{aligned}
A^{+}+B^{+} \longrightarrow 2 B^{+}, & \text {rate }=k_{q} a b, \\
A^{+}+2 B^{+} \longrightarrow 3 B^{+}, & \text {rate }=k_{c} a b^{2}, \\
B^{+}+C^{+} \longrightarrow 2 B^{+}, & \text {rate }=k_{1} b,
\end{aligned}
$$

where $k_{q}, k_{c}, k_{1}$ are the constants and $a, b$ are the concentrations of $A^{+}$and $B^{+}$.

This leads to the dimensionless reactor model

$$
\begin{aligned}
& u_{t}=\delta u_{x x}-\delta \phi u_{x}+\mu-p u v-(1-p) u v^{2}, \\
& v_{t}=v_{x x}-\phi v_{x}+p u v+(1-p) u v^{2}-v,
\end{aligned}
$$

where $u, v, t, x$ are the dimensionless concentrations and time and distance along the reactor, respectively. With the Dirichlet boundary condition

$$
u(t, x)=v(t, x)=0, \quad t \geq 0, x \in \partial \Omega
$$

and an initial condition

$$
\begin{aligned}
& u(0, x)=u_{0}(x), \\
& v(0, x)=v_{0}(x), \\
& x \in \Omega,
\end{aligned}
$$

where $\Omega$ is the bounded set $[0,1]$.

Here, $\delta=D_{A} / D_{B}$ is the radio of diffusion coefficients of substrate $A^{+}$and autocatalyst $B^{+} . \phi$ is a parameter denoting the intensity of the applied electric field. The parameter $p \in$ $[0,1]$ measures the strength of the quadratic step against the cubic step. For $p=1$, the nonlinearity in the kinetics is purely quadratic, while for $p=0$, it is purely cubic.

Satnoianu et al. discussed that diffusion-distributed structures (FDS) are predicted in a wider domain and are more robust than the classical Turing instability patterns. FDS also represent a natural extension of flow-distributed oscillations. Nonlinear bifurcation analysis and numerical simulations in one-dimensional spatial domains show that FDS have much richer solution behavior than Turing structures. Tang and Wang [2] showed a rigorous bifurcation analysis for the model with combination of quadratic and cubic steps by the parameters $p \in[0,1]$ and $\phi=0$. 
For the infinite-dimensional dynamical systems about the chemical model You [3] considered the Brusselator equations; he explored a decomposition technique to show the $\kappa$-contraction of the solution semiflow and proved the existence of a global attractor for the solution semiflow of the Brusselator equation and the Hausdorff dimension and the fractal dimension of the global attractors are finite. You [4] studied the reversible Selkov equations with Dirichlet boundary condition on a bounded domain which is a representative cubic-autocatalytic reaction-diffusion system. He obtained the existence of $\left(L^{2}, H^{1}\right)$ global attractor and the upper semicontinuity of the global attractors in $H^{1}$ product space when the reverse reaction rate tends to zero. For more details about the infinite-dimension dynamics systems about reaction-diffusion models, we can refer to [5-11].

In this paper we will discuss the long-time behavior of the solutions to (2) $-(4)$ for $\phi=0$; that is,

$$
\begin{aligned}
u_{t} & =\delta u_{x x}+\mu-p u v-(1-p) u v^{2}, \\
v_{t} & =v_{x x}+p u v+(1-p) u v^{2}-v, \\
u(t, x) & =v(t, x)=0, \quad t \geq 0, \quad x \in \partial \Omega, \\
u(0, x) & =u_{0}(x), \quad v(0, x)=v_{0}(x), \quad x \in \Omega .
\end{aligned}
$$

We prove the existence of the global attractor by Kuratowski measure. The paper is organized as follows. In Section 2, we prove the existence the weak solution of the system and absorbing property. In Section 3, we give asymptotic compactness of the semiflow by Kuratowski measure and prove the existence of the global attractor. Finally, in Section 4, we give the simulation of the system.

\section{Absorbing Property}

Define the product Hilbert spaces as follows:

$$
\begin{aligned}
H & =\left[L^{2}(\Omega)\right]^{2}, \\
E & =\left[H_{0}^{1}(\Omega)\right]^{2}, \\
V & =\left[H_{0}^{1}(\Omega) \cap H^{2}(\Omega)\right]^{2} .
\end{aligned}
$$

The norm and inner-product of $H$ or the component space $L^{2}(\Omega)$ will be denoted by $\|\cdot\|$ and $\langle\cdot\rangle$, respectively. The norm of $L^{p}(\Omega)$ will be represented by $\|\cdot\|_{L^{p}}$ if $p \neq 2$.

By the poincaré inequality and the homogenous Dirichlet boundary condition there is a constant $\gamma>0$ such that

$$
\|\nabla \phi\|^{2} \geq \gamma\|\phi\|^{2} ; \quad \text { for } \phi \in H_{0}^{1}(\Omega) \text { or } E .
$$

We will take $\|\nabla \phi\|$ to the norm $\|\phi\|_{E}$ of the space $E$.

Using the analytic semigroup generation theorem and Lumer-Phillips theorem, it is easy to check that the densely sectorial operator

$$
A=\left(\begin{array}{cc}
\delta \Delta & 0 \\
0 & \Delta
\end{array}\right): D(A) \longrightarrow H
$$

is the generator of an analytic $C_{0}$-semigroup on the Hilbert space.

By the fact that $H_{0}^{1}(\Omega) \hookrightarrow L^{6}(\Omega) \hookrightarrow L^{4}(\Omega)$ is a continuous embedding, we can verify that the nonlinear mapping

$$
F(u, v)=\left(\begin{array}{c}
\mu-p u v-(1-p) u v^{2} \\
p u v+(1-p) u v^{2}-v
\end{array}\right): E \longrightarrow H
$$

is well defined on $E$ and the mapping $F$ is locally Lipschitz continuous. Let $g(t)=(u, v)$, and then the initial-boundary value problem (5) is formulated into an initial value problem of the abstract evolutionary equation:

$$
\begin{gathered}
\frac{d g}{d t}=A g+F(g), \\
g(0)=g_{0}=\operatorname{Col}\left(u_{0}, v_{0}\right) .
\end{gathered}
$$

A function $g(t, x),(t, x) \in[0, \tau] \times \Omega$ is called a weak solution to the problem of the parabolic evolutionary equation (10), if the following two conditions are satisfied:

(1) $(d / d t)(g, \phi)=(A g, \phi)+(F(g), \phi)$ is satisfied for a.e. $t \in[0, \tau]$ and for any $\phi \in E$.

(2) $g \in L^{2}(0, \tau ; E) \cap C_{w}([0, \tau] ; H)$, where $C_{w}([0, \tau] ; H)$ denote the space of weakly continuous functions on $[0, \tau]$ valued in $H$ such that $g(0)=g_{0}$.

By the Galerkin method, analogous to the arguments in $[3,4]$, we can show the following conclusion about existence of solution.

Lemma 1. For any initial $g_{0} \in H$, there exists a unique local weak solution $(u(t), v(t)), t \in[0, \tau]$ for some $\tau>0$ of the evolutionary equation (10) such that $g$ satisfies

$$
g \in C([0, \tau] ; H) \cap C^{1}((0, \tau) ; H) \cap L^{2}([0, \tau] ; E) .
$$

In order to prove the existence of global attractor, we will investigate the absorbing property of the solution semiflow of problem (5).

Lemma 2. Assume $\delta \gamma>2 / 27$, and then, for any initial data $g_{0} \in H_{+}$, there exists an absorbing set $B_{0} \in H$ for the solution semiflow $\{S(t)\}_{t>0}$ of the Cauchy problem of (5):

$$
B_{0}=\left\{g \in H:\|g\|^{2}<\rho_{0}\right\},
$$

where $\rho_{0}$ is a positive constant independent of initial data. 
Proof. Taking the inner-product of the first equation of (5) with $u(t)$ over $\Omega$, we obtain

$$
\begin{aligned}
& \frac{1}{2} \frac{d}{d t}\|u(t)\|^{2}+\delta\|\nabla u(t)\|^{2} \\
& =\mu \int u d x-p \int u^{2} v d x-(1-p) \int u^{2} v^{2} d x \\
& =\mu \int u d x-\frac{1}{1-p} \int\left(u v+\frac{p(1-p)}{2} u\right)^{2} d x \\
& \quad+\frac{p^{2}(1-p)}{4} \int u^{2} d x \\
& \leq \mu \int u d x+\frac{p^{2}(1-p)}{4} \int u^{2} d x .
\end{aligned}
$$

Let $f(p)=p^{2}(1-p)$, and since $\max _{0 \leq p \leq 1} f(p)=f(2 / 3)=$ $4 / 27$, we have

$$
\begin{aligned}
& \frac{1}{2} \frac{d}{d t}\|u(t)\|^{2}+\delta\|\nabla u(t)\|^{2} \\
& \leq \frac{\mu^{2}|\Omega|}{2 \delta \gamma}+\frac{\delta \gamma}{2}\|u\|^{2}+\frac{1}{27}\|u\|^{2} \\
& \leq \frac{\mu^{2}|\Omega|}{2 \delta \gamma}+\frac{\delta}{2}\|\nabla u\|^{2}+\frac{1}{27 \gamma}\|\nabla u\|^{2} ;
\end{aligned}
$$

that is,

$$
\frac{d}{d t}\|u(t)\|^{2}+\left(\delta-\frac{2}{27 \gamma}\right)\|\nabla u(t)\|^{2} \leq \frac{\mu^{2}|\Omega|}{\delta \gamma} .
$$

Poincáre inequality and Gronwall's inequality yield

$$
\|u(t)\|^{2} \leq e^{-(\delta \gamma-2 / 27) t}\left\|u_{0}\right\|^{2}+\frac{\mu^{2}|\Omega|}{\delta \gamma(\delta \gamma-2 / 27)} .
$$

Let $\rho_{1}=\mu^{2}|\Omega| / \delta \gamma(\delta \gamma-2 / 27)$, and since $\delta>2 / 27 \gamma$, we have

$$
\limsup _{t \rightarrow \infty}\|u(t)\|^{2} \leq \rho_{1} .
$$

Adding two equations in (5) to get an equation for the sum $z(t)=u(t)+v(t)$, one has

$$
z_{t}=z_{x x}+(\delta-1) u_{x x}+\mu-z+u .
$$

Taking the inner-product (18) with $z(t)$ and integrating by parts, we obtain

$$
\begin{aligned}
\frac{1}{2} \frac{d}{d t}\|z\|^{2}+\|\nabla z\|^{2}+\|z\|^{2} \\
=(\delta-1) \int u_{x x} z d x+\int u z d x+\int \mu z d x \\
\leq \frac{1}{2}\|\nabla z\|^{2}+\frac{|\delta-1|^{2}}{2}\|\nabla u\|^{2}+\frac{1}{4}\|z\|^{2}+\|u\|^{2} \\
\quad+\frac{1}{4}\|z\|^{2}+\mu^{2}|\Omega| .
\end{aligned}
$$

Thanks to (16), we have

$$
\begin{aligned}
& \frac{d}{d t}\|z\|^{2}+\|\nabla z\|^{2}+\|z\|^{2} \\
& \leq(\delta-1)^{2}\|\nabla u\|^{2}+2\|u\|^{2}+2 \mu^{2}|\Omega| \\
& \leq(\delta-1)^{2}\|\nabla u\|^{2}+2 e^{-(\delta \gamma-1 / 27) t}\left\|u_{0}\right\|^{2} \\
& \quad+2\left(1+\frac{1}{\delta \gamma(\delta \gamma-2 / 27)}\right) \mu^{2}|\Omega| .
\end{aligned}
$$

Let $C_{0}(t)=2 e^{-(\delta \gamma-2 / 27) t}\left\|u_{0}\right\|^{2}+2(1+1 / \delta \gamma(\delta \gamma-2 / 27)) \mu^{2}|\Omega|$, and then we have

$$
\frac{d}{d t}\|z\|^{2}+\|\nabla z\|^{2}+\|z\|^{2} \leq(\delta-1)^{2}\|\nabla u\|^{2}+C_{0}(t)
$$

that is,

$$
\frac{d}{d t}\left(e^{t}\|z\|^{2}\right) \leq(\delta-1)^{2} e^{t}\|\nabla u\|^{2}+C_{0}(t) e^{t}
$$

integrating (22) from 0 to $t$, and then

$$
\begin{aligned}
\|z\|^{2} \leq & e^{-t}\left\|z_{0}\right\|^{2}+(\delta-1)^{2} \int_{0}^{t} e^{-(t-\tau)}\|\nabla u\|^{2} d \tau \\
& +C_{1}(t)
\end{aligned}
$$

where

$$
\begin{aligned}
& C_{1}(t) \\
& \begin{aligned}
= & e^{-t} \int_{0}^{t} e^{[1-(\delta \gamma-2 / 27)] \tau}\left\|u_{0}\right\|^{2} d \tau \\
& +\left(2+\frac{2}{\delta \gamma(\delta \gamma-2 / 27)}\right) \mu^{2}|\Omega|,
\end{aligned} \\
& 2 e^{-t} \int_{0}^{t} e^{[1-(\delta \gamma-2 / 27)] \tau}\left\|u_{0}\right\|^{2} d \tau \\
& \leq \begin{cases}\frac{2}{1-(\delta \gamma-2 / 27)} e^{-(\delta \gamma-2 / 27) t}\left\|u_{0}\right\|^{2}, & 1-\left(\delta \gamma-\frac{2}{27}\right)>0 \\
2 e^{-t} t\left\|u_{0}\right\|^{2}, & 1-\left(\delta \gamma-\frac{2}{27}\right)=0 \\
\frac{2}{|1-(\delta \gamma-2 / 27)|} e^{-t}\left\|u_{0}\right\|^{2}, & 1-\left(\delta \gamma-\frac{2}{27}\right)<0 .\end{cases}
\end{aligned}
$$

Now we treat the term $\int_{0}^{t} e^{-(t-\tau)}\|\nabla u\|^{2} d \tau$ in (23); multiplying (15) by $e^{t}$ and integrating from 0 to $t$, we get

$$
\begin{aligned}
& \int_{0}^{t}\left(e^{\tau} \frac{d}{d \tau}\|u\|^{2}\right) d \tau+\left(\delta-\frac{2}{27 \gamma}\right) \int_{0}^{t} e^{\tau}\|\nabla u\|^{2} d \tau \\
& \quad \leq \frac{\mu^{2}|\Omega|}{\delta \gamma} e^{t}
\end{aligned}
$$


and, by (16), we have

$$
\begin{aligned}
(\delta & \left.-\frac{2}{27 \gamma}\right) \int_{0}^{t} e^{\tau}\|\nabla u\|^{2} d \tau \\
& \leq \frac{\mu^{2}|\Omega|}{\delta \gamma} e^{t}-\int_{0}^{t}\left(e^{\tau} \frac{d}{d \tau}\|u\|^{2}\right) d \tau \\
& \leq \frac{\mu^{2}|\Omega|}{\delta \gamma} e^{t}-\left(e^{t}\|u\|^{2}-\left\|u_{0}\right\|^{2}\right)+\int_{0}^{t} e^{\tau}\|u\|^{2} d \tau \\
& \leq \frac{\mu^{2}|\Omega|}{\delta \gamma} e^{t}+\left\|u_{0}\right\|^{2} \\
& +\int_{0}^{t} e^{\tau}\left(e^{-(\delta \gamma-2 / 27) \tau}+\frac{\mu^{2}|\Omega|}{\delta \gamma(\delta \gamma-2 / 27)}\right) d \tau \\
\leq & \frac{\mu^{2}|\Omega|}{\delta \gamma} e^{t}+\left\|u_{0}\right\|^{2}+\frac{\mu^{2}|\Omega|}{\delta \gamma(\delta \gamma-2 / 27)} e^{t}+C_{2}(t),
\end{aligned}
$$

where

$$
\begin{aligned}
& C_{2}(t)=\int_{0}^{t} e^{[1-(\delta \gamma-2 / 27)] \tau}\left\|u_{0}\right\|^{2} d \tau \\
& \leq \begin{cases}\frac{1}{1-(\delta \gamma-2 / 27)} e^{[1-(\delta \gamma-2 / 27)] t}\left\|u_{0}\right\|^{2}, & 1-\left(\delta \gamma-\frac{2}{27}\right)>0 \\
t\left\|u_{0}\right\|^{2}, & 1-\left(\delta \gamma-\frac{2}{27}\right)=0 \\
\frac{1}{|1-(\delta \gamma-2 / 27)|}\left\|u_{0}\right\|^{2}, & 1-\left(\delta \gamma-\frac{2}{27}\right)<0 .\end{cases}
\end{aligned}
$$

Substituting (26) into (23), we obtain

$$
\begin{aligned}
\|z\|^{2} \leq & e^{-t}\left\|u_{0}+v_{0}\right\|^{2}+\frac{\mu^{2}(\delta-1)^{2}|\Omega|}{\delta(\delta \gamma-2 / 27)} \\
& +\frac{(\delta-1)^{2}}{\delta-2 / 27 \gamma} e^{-t}\left\|u_{0}\right\|^{2}+\frac{\mu^{2}(\delta-1)^{2}|\Omega|}{\delta(\delta \gamma-2 / 27)^{2}} \\
& +\frac{(\delta-1)^{2}}{\delta-2 / 27 \gamma} e^{-t} C_{2}(t)+C_{1}(t) .
\end{aligned}
$$

Noticing that $e^{-t} C_{2}(t) \rightarrow 0, C_{1}(t) \rightarrow 0$, as $t \rightarrow+\infty$, therefore we conclude that

$$
\limsup _{t \rightarrow+\infty}\|z\|^{2} \leq \frac{\mu^{2}(\delta-1)^{2}|\Omega|}{\delta(\delta \gamma-2 / 27)}+\frac{\mu^{2}(\delta-1)^{2}|\Omega|}{\delta(\delta \gamma-2 / 27)^{2}} .
$$

Let $\rho_{2}=\mu^{2}(\delta-1)^{2}|\Omega| / \delta(\delta \gamma-2 / 27)+\mu^{2}(\delta-1)^{2}|\Omega| / \delta(\delta \gamma-$ $2 / 27)^{2}$, which is independent of any initial data, and then we get

$$
\limsup _{t \rightarrow+\infty}\|z\|^{2} \leq \rho_{2}
$$

Combining (17) and (30), we can deduce that for any initial data $g_{0}=\left(u_{0}, v_{0}\right) \in H$ the solution satisfies

$$
\begin{aligned}
\limsup _{t \rightarrow+\infty}\|g\|^{2} & =\limsup _{t \rightarrow+\infty}\left(\|u\|^{2}+\|v\|^{2}\right) \\
& =\limsup _{t \rightarrow+\infty}\left(\|z-u\|^{2}+\|u\|^{2}\right) \\
& \leq \limsup _{t \rightarrow+\infty}\left(3\|u\|^{2}+2\|z\|^{2}\right) \leq 3 \rho_{1}+2 \rho_{2} \\
& :=\rho_{0} .
\end{aligned}
$$

So set $B_{0}$ in (12) is the absorbing set for the solution semiflow to problem (5). Then proof is completed.

\section{The Existence of Global Attractor}

In this section we will prove that the solution semiflow $\{S(t)\}_{t>0}$ of (5) has a global attractor. According the existence of criterion in $[6,12]$, we just need to prove that the semiflow $\{S(t)\}_{t>0}$ is asymptotic compactness. Firstly, the following proposition is about the uniform Gronwall's inequality which is a basic tool in the analysis of asymptotics.

Lemma 3. Let $\beta, \zeta$ and $h$ be nonnegative function in $L_{\text {Loc }}^{1}(0, \infty, R)$. Assume that $\beta$ is absolutely continuous on $[0,+\infty)$ and the following differential inequality is satisfied:

$$
\frac{d \beta}{d t} \leq \zeta \beta+h
$$

If there is a finite time $t_{1}>0$ and some $r>0$, such that

$$
\begin{aligned}
& \int_{t}^{t+r} \zeta(\tau) d \tau \leq A, \\
& \int_{t}^{t+r} \beta(\tau) d \tau \leq B, \\
& \int_{t}^{t+r} h(\tau) d \tau \leq C,
\end{aligned}
$$

for any $t>t_{1}$, where $A, B$, and $C$ are positive constants then

$$
\beta(t) \leq\left(\frac{B}{r}+C\right) e^{A},
$$

for any $t>t_{1}+r$.

Lemma 4. There is a constant $M_{0}>0$, such that

$$
\int_{t}^{t+1}\left(\|\nabla u(\tau)\|^{2}+\|\nabla v(\tau)\|^{2}\right) d \tau \leq M_{0}
$$

for any $t>T_{0}, g_{0} \in B_{0}$, where $T_{0}=T_{0}\left(B_{0}\right)$ is a positive constant depending only on the absorbing set $B_{0}$.

Proof. From (15) we have

$$
\left(\delta-\frac{2}{27 \gamma}\right) \int_{t}^{t+1}\|\nabla u(\tau)\|^{2} d \tau \leq\|u(t)\|^{2}+\frac{\mu^{2}|\Omega|}{\delta \gamma} .
$$


Since the absorbing set $B_{0}$ absorbs itself, there exists $T_{0}>0$ such that $S(t) B_{0} \subset B_{0}$ for any $t>T_{0}$, so we obtain

$$
\begin{aligned}
\int_{t}^{t+1}\|\nabla u(\tau)\|^{2} d \tau & \leq \frac{1}{\delta-2 / 27 \gamma}\left(\rho_{0}+\frac{\mu^{2}|\Omega|}{\delta \gamma}\right) \\
:=M_{1} &
\end{aligned}
$$

From (21) and (37), we get

$$
\begin{aligned}
\int_{t}^{t+1} & \|\nabla z(\tau)\|^{2} d \tau \\
\leq & (\delta-1)^{2} \int_{t}^{t+1}\|\nabla u(\tau)\|^{2} d \tau+\int_{t}^{t+1} C_{0}(\tau) d \tau \\
& +\|z(t)\|^{2} \\
\leq & (\delta-1)^{2} M_{1}+2\left(1+\frac{1}{\delta \gamma(\delta \gamma-2 / 27)}\right) \mu^{2}|\Omega| \\
& +\rho_{0}:=M_{2} .
\end{aligned}
$$

Since $v(t)=z(t)-u(t)$, it follows from (37) and (38); we obtain for any $t>T_{0}$

$$
\begin{aligned}
& \int_{t}^{t+1}\left(\|\nabla u(\tau)\|^{2}+\|\nabla v(\tau)\|^{2}\right) d \tau \\
& \quad= \int_{t}^{t+1}\left(\|\nabla u(\tau)\|^{2}+\|\nabla(z(\tau)-u(\tau))\|^{2}\right) d \tau \\
& \quad \leq 3 M_{1}+2 M_{2}:=M_{0} .
\end{aligned}
$$

The proof is completed.

Recall the definition of the Kuratowski measure of noncompactness for bounded sets in a Banach space $X[13,14]$ :

$K(B)=\inf \{\delta:$

$B$ has a finite cover by open sets in $X$ of diameters

$$
<\delta\} \text {. }
$$

A semiflow $\{S(t)\}_{t \geq 0}$ on a complete metric space $X$ is called $\kappa$-contracting if, for every bounded subset $B$ in $X$, one has

$$
\lim _{t \rightarrow \infty} K(S(t) B)=0 .
$$

Lemma 5. Let $\{S(t)\}_{t \geq 0}$ be a semiflow on a Banach space $X$, if the following conditions are satisfied:

(1) $\{S(t)\}_{t \geq 0}$ has a bounded absorbing set in $X$.

(2) $\{S(t)\}_{t \geq 0}$ is $\kappa$-contracting.

Then $\{S(t)\}_{t \geq 0}$ is asymptotically compact and there exists a global attractor $\mathscr{A}$ in $X$.

Lemma 6 (see [4]). Let $\{S(t)\}_{t \geq 0}$ be a semiflow on $H$, and there exists a global attractor $\mathscr{A}$ in $H$ for this semiflow, if and only if the following conditions are satisfied:
(1) There exists a bounded absorbing set $B_{0}$ in $H$ for this semiflow.

(2) For any $\varepsilon>0$, there are positive constants $M=M(\varepsilon)$ and $T=T(\varepsilon)$ such that

$$
\begin{aligned}
& \int_{\Omega\left(\left|S(t) g_{0}\right| \geq M\right)}\left|S(t) g_{0}\right|^{2} d x<\varepsilon, \quad \forall t \geq T, g_{0} \in B_{0}, \\
& K\left(\left(S(t) g_{0}\right)_{\Omega\left(\left|S(t) g_{0}\right| \leq M\right)}\right) \longrightarrow 0, \quad \text { as } t \longrightarrow \infty,
\end{aligned}
$$

where $\left(S(t) g_{0}\right)_{\Omega\left(\left|S(t) g_{0}\right| \leq M\right)}=\left\{\left(S(t) g_{0}\right)(\cdot) \theta_{M}\left(\cdot ; t, g_{0}\right)\right.$, for $g_{0} \epsilon$ $\left.B_{0}\right\}$, and $\theta_{M}\left(\cdot ; t, g_{0}\right)$ is the characteristic function of the subset $\Omega\left(\left|S(t) g_{0}\right| \leq M\right)$.

Lemma 7. For any $\varepsilon>0$, there exist positive constants $M_{3}(\varepsilon)$ and $T_{3}(\varepsilon)$, such that the u-component of the solution to problem (5) satisfies

$$
\int_{\Omega\left(|u(t)| \geq M_{3}\right)}|u(t)|^{2} d x<\varepsilon, \quad \forall t \geq T_{3}, \quad g_{0} \in B_{0} .
$$

Proof. Since $B_{0}$ is a bounded absorbing set, there exist $T_{0}\left(B_{0}\right)>0$ and $K_{0}>0$ such that

$$
\|u(t)\|^{2} \leq \rho_{0}, \quad \text { for } t \geq T_{0}, g_{0} \in B_{0} .
$$

Hence we have

$$
M^{2} m(\Omega(|u(t)|>M)) \leq \int_{\Omega(|u(t)|>M)}|u(t)|^{2} d x \leq \rho_{0},
$$

and hence there exist $M(\varepsilon)$ such that

$$
m(\Omega(|u(t)|>M)) \leq \frac{K_{0}}{M^{2}}<\frac{\varepsilon}{2}
$$

Taking the inner-product (5) with $(u(t)-M)_{+}$where $M$ is given in (46) and

$$
(u(t)-M)_{+}= \begin{cases}u(t)-M, & u(t) \geq M \\ 0, & u(t)<M\end{cases}
$$

we obtain

$$
\begin{aligned}
& \frac{1}{2} \frac{d}{d t}\left\|(u-M)_{+}\right\|^{2}+\delta \int_{\Omega(u(t)>M)}\left|\nabla(u-M)_{+}\right|^{2} d x \\
& \leq \mu \int_{\Omega(u(t)>M)}(u-M)_{+} d x \\
& \leq \frac{\delta \gamma}{2} \int_{\Omega(u(t)>M)}(u-M)_{+}^{2} d x \\
& \quad+\frac{\mu^{2}}{2 \delta \gamma} m(\Omega(u(t)>M)) \\
& \leq \frac{\delta}{2} \int_{\Omega(u(t)>M)}\left|\nabla(u-M)_{+}\right|^{2} d x+\frac{\mu^{2}}{2 \delta \gamma} \varepsilon .
\end{aligned}
$$


It follows that

$$
\begin{aligned}
& \frac{d}{d t}\left\|(u-M)_{+}\right\|^{2}+\delta \int_{\Omega(u(t)>M)}\left|\nabla(u-M)_{+}\right|^{2} d x \\
& \quad \leq \frac{\mu^{2}}{\delta \gamma} \varepsilon .
\end{aligned}
$$

By Poincáre inequality, we have

$$
\begin{aligned}
& \frac{d}{d t}\left\|(u-M)_{+}\right\|^{2}+\delta \gamma \int_{\Omega(u(t)>M)}\left|(u-M)_{+}\right|^{2} d x \\
& \quad \leq \frac{\mu^{2}}{\delta \gamma} \varepsilon .
\end{aligned}
$$

By Gronwall's lemma, we obtain

$$
\left\|(u-M)_{+}\right\|^{2} \leq e^{-\delta \gamma t}\left\|\left(u_{0}-M\right)_{+}\right\|^{2}+\frac{\mu^{2}}{(\delta \gamma)^{2}} \varepsilon .
$$

Thus there exist $T_{1}(\varepsilon, M)>T_{0}$, such that, for any $t>T_{1}$ and any $g_{0} \in B_{0}$, one has

$$
\left\|(u-M)_{+}\right\|^{2} \leq \frac{2 \mu^{2}}{(\delta \gamma)^{2}} \varepsilon .
$$

Symmetrically, we can prove that there exists $T_{2}(\varepsilon, M)>T_{0}$ such that for any $t<-T_{2}$ and any $g_{0} \in B_{0}$ one has

$$
\left\|(u+M)_{-}\right\|^{2} \leq \frac{2 \mu^{2}}{(\delta \gamma)^{2}} \varepsilon,
$$

where

$$
(u(t)+M)_{-}= \begin{cases}u(t)+M, & u(t) \leq-M \\ 0, & u(t)>-M .\end{cases}
$$

Therefore, let $T_{3}=\max \left\{T_{1}, T_{2}\right\}$, for any $t>T_{3}$ and $g_{0} \in B_{0}$, and there hold

$$
\int_{\Omega(|u(t)|>M)}(|u|-M)^{2} d x \leq \frac{2 \mu^{2}}{(\delta \gamma)^{2}} \varepsilon .
$$

Next we can deduce that there exists a positive integer $k$, such that

$$
\int_{\Omega(|u(t)|>k M)}|u|^{2} d x \leq \frac{8 \mu^{2}}{(\delta \gamma)^{2}} \varepsilon .
$$

Indeed it follows that

$$
\begin{aligned}
\int_{\Omega(|u(t)|>k M)}|u|^{2} d x \leq & 2 \int_{\Omega(|u(t)|>M)}(|u|-M)^{2} d x \\
& +2 M^{2} m(\Omega(|u(t)| \geq k M)) \\
\leq & \frac{4 \mu^{2}}{(\delta \gamma)^{2}} \varepsilon+2 M^{2} \frac{K_{0}}{k^{2} M^{2}} \\
= & \frac{4 \mu^{2}}{(\delta \gamma)^{2}} \varepsilon+\frac{2 K_{0}}{k^{2}} \leq \frac{8 \mu^{2}}{(\delta \gamma)^{2}} \varepsilon
\end{aligned}
$$

Since $m(\Omega(|u(t)| \geq k M)) \leq K_{0} / k^{2} M^{2}$, where $K_{0} / M^{2}<\varepsilon / 2$ for a sufficiently large integer $k$, (43) holds, with $M_{3}=k M$, $T_{3}=\max \left\{T_{1}, T_{2}\right\}$ for any $g_{0} \in B_{0}$. The proof is completed.

Lemma 8. Let $B_{0}$ be the bounded absorbing set of solution semiflow $\{S(t)\}_{t \geq 0}$ to problem (5), and then, for any given $M_{4}>$ 0 , it hold that

$$
K\left(P_{u}\left(S(t) B_{0}\right)_{\Omega\left(|u(t)|<M_{4}\right)}\right) \longrightarrow 0, \quad \text { as } t \longrightarrow \infty
$$

where $P_{u}: H \rightarrow L^{2}(\Omega)$ is the orthogonal projection from the product Hilbert space $H$ onto the first component space associated with the u-component.

Proof. Taking the inner-product (5) with $-\Delta u(t)$ over the set $\Omega_{1}=\Omega\left(|u(t)|<M_{4}\right)$,

$$
\begin{aligned}
& \frac{1}{2} \frac{d}{d t}\|\nabla u\|_{\Omega_{1}}^{2}+\delta\|\Delta u\|_{\Omega_{1}}^{2}=-\mu \int_{\Omega_{1}} \Delta u d x \\
& \quad+p \int_{\Omega_{1}} u v \Delta u d x+(1-p) \int_{\Omega_{1}} u v^{2} \Delta u d x \\
& \quad \leq \frac{\mu^{2}|\Omega|}{2 \delta}+\frac{\delta}{2}\|\Delta u\|_{\Omega_{1}}^{2}-p \int_{\Omega_{1}} \nabla(u v) \nabla u d x-(1 \\
& \quad-p) \int_{\Omega_{1}} \nabla\left(u v^{2}\right) \nabla u d x \leq \frac{\mu^{2}|\Omega|}{2 \delta}+\frac{\delta}{2}\|\Delta u\|_{\Omega_{1}}^{2} \\
& \quad-p \int_{\Omega_{1}} v|\nabla u|^{2} d x-p \int_{\Omega_{1}} u \nabla u \nabla v d x-(1-p) \\
& \quad\left[\int_{\Omega_{1}} v^{2}|\nabla u|^{2} d x\right. \\
& \left.\quad+2 \int_{\Omega_{1}} u v \nabla u \nabla v d x+\int_{\Omega_{1}}|\nabla v|^{2} u^{2} d x\right]+(1-p) \\
& \quad+\frac{\mu^{2}|\Omega|}{2 \delta}+\frac{\delta}{2}\|\Delta u\|_{\Omega_{1}}^{2}+\frac{\delta \gamma}{4} \int_{\Omega_{1}}|\nabla u|^{2} d x+\left(\frac{p^{2}}{\delta \gamma}\right. \\
& \quad+\left.p v\right|_{\Omega_{1}} ^{2} u \int^{2} d x \leq \frac{\mu^{2}|\Omega|}{2 \delta}+\frac{\delta}{2}\|\Delta u\|_{\Omega_{1}}^{2} \\
& \quad+\int_{\Omega_{1}}|\nabla v|^{2} u^{2} d x
\end{aligned}
$$

so we obtain

$$
\begin{aligned}
& \frac{d}{d t}\|\nabla u\|_{\Omega_{1}}^{2}+\frac{\delta \gamma}{2}\|\nabla u\|_{\Omega_{1}}^{2} \\
& \quad \leq\left(\frac{p^{2}}{\delta \gamma}+1-p\right) \int_{\Omega_{1}}|\nabla v|^{2} u^{2} d x+\frac{\mu^{2}|\Omega|}{\delta},
\end{aligned}
$$


and since $|u(t)|<M_{4}$, we have

$$
\begin{aligned}
& \frac{d}{d t}\|\nabla u\|_{\Omega_{1}}^{2}+\frac{\delta \gamma}{2}\|\nabla u\|_{\Omega_{1}}^{2} \\
& \quad \leq\left(\frac{p^{2}}{\delta \gamma}+1-p\right) M_{4}^{2} \int_{\Omega_{1}}|\nabla v|^{2} d x+\frac{\mu^{2}\left|\Omega_{1}\right|}{\delta} .
\end{aligned}
$$

It follows that $\|\nabla v\|^{2} \leq\|\nabla z\|^{2}+\|\nabla u\|^{2}$. By Lemma 4, we get that there exits $T_{0}=T_{0}\left(B_{0}\right)>0$ such that for all $t>T_{0}$

$$
\int_{t}^{t+1}\|\nabla v\|^{2} d \tau \leq \int_{t}^{t+1}\left(\|\nabla z\|^{2}+\|\nabla u\|^{2}\right) d \tau \leq M_{0} .
$$

Combining results in Lemma 3, (61), and (62), we conclude that there exist $\rho_{3}>0, T_{4}>T_{3}$ such that

$$
\|\nabla u\|_{\Omega_{1}}^{2} \leq \rho_{3} \quad \forall t>T_{4}, g_{0} \in B_{0} .
$$

Inequality (63) is uniform constant depending on the absorbing set $B_{0}$ and the given $M_{4}$ and shows that, for any fixed $t>T_{4}, P_{u}\left(S(t) B_{0}\right)_{\Omega\left(|u| \leq M_{4}\right)}$ is bounded in $H_{0}^{1}(\Omega)$. Due to the compact embedding $H_{0}^{1}(\Omega) \hookrightarrow L^{2}(\Omega)$, it turns out that, for any fixed $t>T_{4}, P_{u}\left(S(t) B_{0}\right)_{\Omega\left(|u| \leq M_{4}\right)}$ is a precompact set in $L^{2}(\Omega)$, so we have $K\left(P_{u}\left(S(t) B_{0}\right)_{\Omega\left(|u(t)|<M_{4}\right)}\right)=0$, for $t \geq T_{4}$ in the space $L^{2}(\Omega)$. The proof is completed.

Lemma 9. For any $\varepsilon>0$, there exist positive constants $M_{5}(\varepsilon)$ and $T_{5}(\varepsilon)$, such that the $v$-component of the solution semiflow of problem (5) satisfies

$$
\int_{\Omega\left(|u(t)| \geq M_{5}\right)}|v(t)|^{2} d x<\varepsilon, \quad \text { for any } t \geq T_{5}, \quad g_{0} \in B_{0} .
$$

Proof. Taking the inner-product (18) with $z(t)$ over $\Omega_{2}=$ $\left\{|u(t)|>M_{3}\right\}$, we get

$$
\begin{aligned}
& \frac{1}{2} \frac{d}{d t}\|z\|_{\Omega_{2}}^{2}+\|\nabla z\|_{\Omega_{2}}^{2}+\|z\|_{\Omega_{2}}^{2}(\delta-1) \int_{\Omega_{2}} \Delta u z d x+\int_{\Omega_{2}}(\mu+u) z d x \\
& \leq \frac{1}{2}\|\nabla z\|_{\Omega_{2}}^{2}+\frac{(\delta-1)^{2}}{2}\|\nabla u\|_{\Omega_{2}}^{2}+\frac{1}{2}\|z\|_{\Omega_{2}}^{2} \\
&+\frac{1}{2}\|\mu+u\|_{\Omega_{2}}^{2} .
\end{aligned}
$$

We obtain

$$
\begin{aligned}
& \frac{d}{d t}\|z\|_{\Omega_{2}}^{2}+\|\nabla z\|_{\Omega_{2}}^{2}+\|z\|_{\Omega_{2}}^{2} \\
& \quad \leq(\delta-1)^{2}\|\nabla u\|_{\Omega_{2}}^{2}+2\|u\|_{\Omega_{2}}^{2}+2 \mu^{2}|\Omega| .
\end{aligned}
$$

Integrating both sides of the above inequality from 0 to $t$, we have

$$
\begin{gathered}
\|z\|_{\Omega_{2}}^{2} \leq e^{-t}\left\|z_{0}\right\|_{\Omega_{2}}^{2}+(\delta-1)^{2} e^{-t} \int_{0}^{t} e^{\tau}\|\nabla u\|_{\Omega_{2}}^{2} d \tau \\
+e^{-t} \int_{0}^{t} e^{\tau}\left(2\|u\|_{\Omega_{2}}^{2}+2 \mu^{2}|\Omega|\right) d \tau .
\end{gathered}
$$

Using inequality (26), the right hand of (67) can be estimated as follows:

$$
\begin{gathered}
e^{-t}\left\|z_{0}\right\|_{\Omega_{2}}^{2}+e^{-t} \int_{0}^{t} e^{\tau}\left(2\|u\|_{\Omega_{2}}^{2}+2 \mu^{2}\left|\Omega_{2}\right|\right) d \tau<\frac{\varepsilon}{2}, \\
(\delta-1)^{2} e^{-t} \int_{0}^{t} e^{\tau}\|\nabla u\|_{\Omega_{2}}^{2} d \tau \\
<(\delta-1)^{2}\left|\Omega_{2}\right|+(\delta-1)^{2} C_{1}(t)\left\|u_{0}\right\|_{\Omega_{2}}<\frac{\varepsilon}{2},
\end{gathered}
$$

for all $M>M_{3}, t>T_{3}$, we find that there exist $T_{2}=$ $\max \left\{T_{0}, T_{1}\right\}, M_{2}$ such that

$$
\|z\|_{\Omega_{2}}^{2}<\varepsilon, \quad \text { for } t>T_{3}, \quad M>M_{3}
$$

Combining (43) and (69), we have that there exist positive $M_{5}>M_{3}, T_{5}>T_{3}$ such that

$$
\begin{array}{r}
\int_{\Omega\left(|u(t)| \geq M_{5}\right)}|v(t)|^{2} d x<2\left(\|u\|_{\Omega_{1}}^{2}+\|z\|_{\Omega_{2}}^{2}\right)<\varepsilon, \\
\text { for any } t \geq T_{5}, \quad g_{0} \in B_{0} .
\end{array}
$$

The proof is completed.

Lemma 10. Let $B_{0}$ be the bounded absorbing set of solution semiflow $\{S(t)\}_{t \geq 0}$ to problem (2)-(4), and then, for any given $M_{4}>0$, it holds that

$$
K\left(P_{v}\left(S(t) B_{0}\right)_{\Omega\left(|u(t)|<M_{4}\right)}\right) \longrightarrow 0, \quad \text { as } t \longrightarrow \infty
$$

where $P_{v}: H \rightarrow L^{2}(\Omega)$ is the orthogonal projection from the product Hilbert space $H$ onto the first component space associated with the $v$-component.

Proof. Taking the inner-product (5) with $-\Delta v(t)$ over the set $\Omega_{1}$, we have

$$
\begin{aligned}
& \frac{1}{2} \frac{d}{d t}\|\nabla v\|_{\Omega_{1}}^{2}+\|\Delta v\|_{\Omega_{1}}^{2} \\
& =-p \int_{\Omega_{1}} u v \Delta v d x-(1-p) \int_{\Omega_{1}} u v^{2} \Delta v d x \\
& \quad+\int_{\Omega_{1}} v \Delta v d x \\
& \leq\left(p M_{4}+1\right) \int_{\Omega_{1}} v|\Delta v| d x \\
& \quad+(1-p) M_{4} \int_{\Omega_{1}} v^{2} \nabla v d x
\end{aligned}
$$




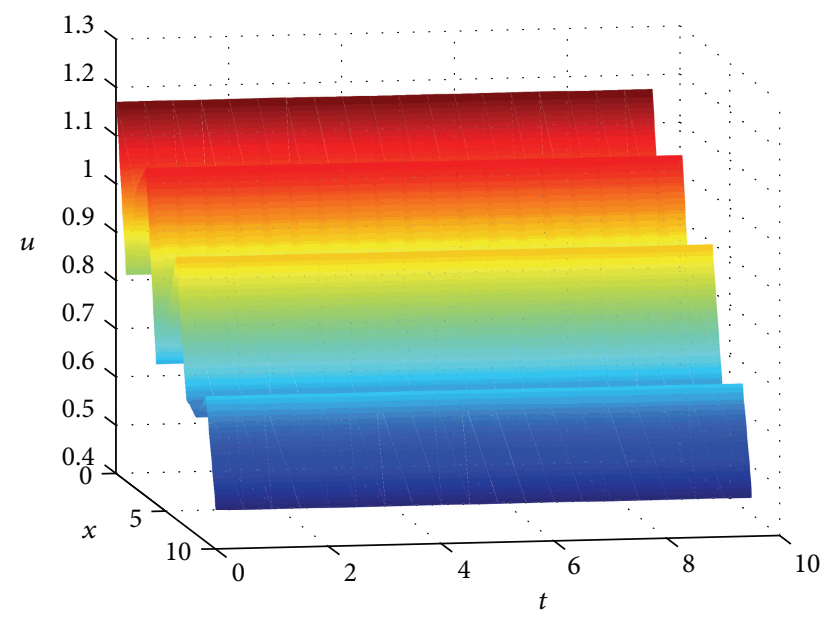

(a)

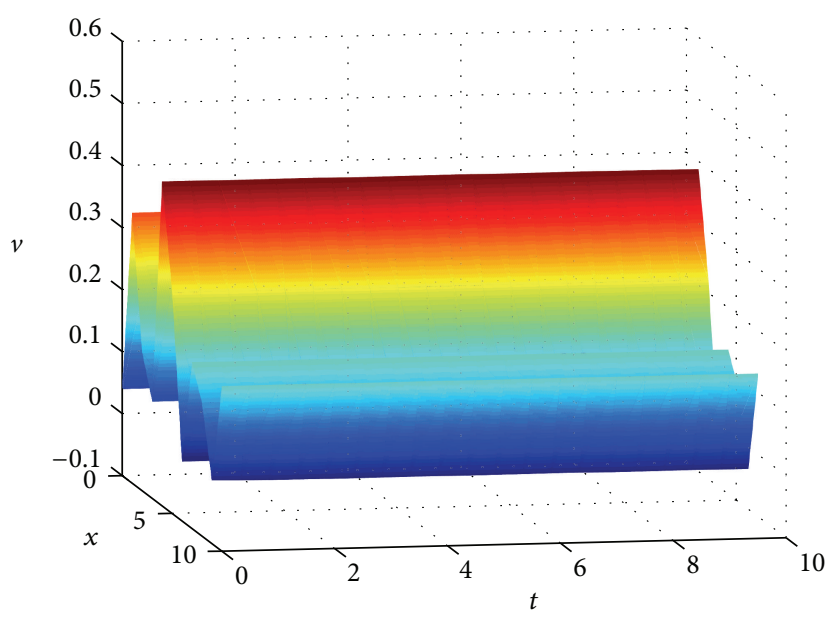

(b)

FIGURE 1: Numerical simulation for (5) with $u_{0}=\cos (x / 10), v_{0}=\cos (x)$.

$$
\begin{aligned}
\leq & \frac{1}{2}\|\Delta v\|_{\Omega_{1}}^{2}+\frac{\left(p M_{4}+1\right)^{2}}{2}\|v\|_{\Omega_{1}}^{2}+\frac{1}{2}\|\Delta v\|_{\Omega_{1}}^{2} \\
& +\frac{(1-p)^{2} M_{4}^{2}}{2} \int_{\Omega_{1}} v^{4} d x \\
\leq & \|\Delta v\|_{\Omega_{1}}^{2}+\frac{\left(p M_{4}+1\right)^{2}}{2} \rho_{0} \\
& +\frac{(1-p)^{2} M_{4}^{2}}{2}\|v\|_{L^{4}\left(\Omega_{1}\right)}^{4} \\
\leq & \|\Delta v\|_{\Omega_{1}}^{2}+\frac{\left(p M_{4}+1\right)^{2}}{2} \rho_{0} \\
& +\frac{(1-p)^{2} M_{4}^{2}}{2 \gamma^{2}}\|\nabla v\|_{\Omega_{1}}^{4},
\end{aligned}
$$

and, thus, it follows that

$$
\begin{aligned}
\frac{d}{d t}\|\nabla v\|_{\Omega_{1}}^{2} \leq & \frac{\left(p M_{4}+1\right)^{2}}{2} \rho_{0} \\
& +\frac{(1-p)^{2} M_{4}^{2}}{2 \gamma^{2}}\|\nabla v\|_{\Omega_{1}}^{2}\|\nabla v\|_{\Omega_{1}}^{2},
\end{aligned}
$$

for $t>T_{0}$. In view of (62)

$$
\frac{(1-p)^{2} M_{4}^{2}}{2 \gamma^{2}} \int_{t}^{t+1}\|\nabla v\|_{\Omega_{1}}^{2} d \tau \leq M_{4} \frac{(1-p)^{2} M_{4}^{2}}{2 \gamma^{2}},
$$

and, applying Lemma 3, there exist $\rho_{4}>0, T_{6}>T_{4}$ such that

$$
\|\nabla v\|_{\Omega_{1}}^{2} \leq \rho_{3} \quad \forall t>T_{6}
$$

it shows that $P_{v}\left(S(t) B_{0}\right)_{\Omega\left(|u(t)|<M_{4}\right)}$ is bounded set in $H_{0}^{1}(\Omega)$ for any $t>T_{6}$, so that $P_{v}\left(S(t) B_{0}\right)_{\Omega\left(|u(t)|<M_{4}\right)}$ is a precompact set in $L^{2}(\Omega)$. It is $K\left(P_{v}\left(S(t) B_{0}\right)_{\Omega\left(|u(t)|<M_{4}\right)}\right)=0$ in $L^{2}(\Omega)$ for $t>T_{6}$. The proof is completed.
Theorem 11. If $\delta>2 / 27 \gamma, 0 \leq p \leq 1$, there exists a global attractor $\mathscr{A}$ in $H$ for the solution semiflow $\{S(t)\}_{t \geq 0}$ generated by problem (5).

Proof. By Lemma 2, we have $\{S(t)\}_{t \geq 0}$ that has a bounded absorbing set $B_{0}$ in $H$ and by Lemmas 8 and 10 we have that the conditions in Lemma 4 are satisfied. So by Lemma 4 there exists a global attractor $\mathscr{A}$ in $H$ for the solution semiflow $\{S(t)\}_{t>0}$ generated by problem (5). The proof is completed.

\section{Simulation}

In this section, using the finite difference method to (5), the discrete equation of (5) was obtained as follows:

$$
\begin{aligned}
\frac{u_{i}^{n+1}-u_{i}^{n}}{\Delta t}= & \frac{\delta\left(u_{i-1}^{n}+u_{i+1}^{n}-2 u_{i}^{n}\right)}{h^{2}}+\mu-p u_{i}^{n} v_{i}^{n} \\
& -(1-p) u_{i}^{n}\left(v_{i}^{n}\right)^{2}, \\
\frac{v_{i}^{n+1}-v_{i}^{n}}{\Delta t}= & \frac{\delta\left(v_{i-1}^{n}+v_{i+1}^{n}-2 v_{i}^{n}\right)}{h^{2}}+p u_{i}^{n} v_{i}^{n} \\
& +(1-p) u_{i}^{n}\left(v_{i}^{n}\right)^{2}-v_{i}^{n} .
\end{aligned}
$$

Here $h$ is space stepsize, $\Delta t$ is time stepsize, and $u_{i}^{n}$ is a numerical approximation to $u\left(x_{j}, t_{n}\right)$ at $\left(x_{j}, t_{n}\right)=(j h, n \Delta t)$. Then we apply Euler's Method with the Dirichlet boundary condition to solve the discrete equation (5). In what follows, we always set space stepsize $h=1$ and time stepsize $\Delta t=0.5$ to simulate the problem under different conditions.

Now we set the parameters $\delta=1, \mu=0.5, p=0.1$, and let initial data $u_{0}(x)=\cos (x / 10), v_{0}(x)=\cos (x)$. The numerical results are shown in Figure 1. These plots indicate that the global attractor in Section 3 is complicated and consist of many period trajectories. In the meanwhile, when we let initial data $u_{0}(x)=\cos (x / 10) v_{0}(x)=\cos (x / 10)$, the plots 


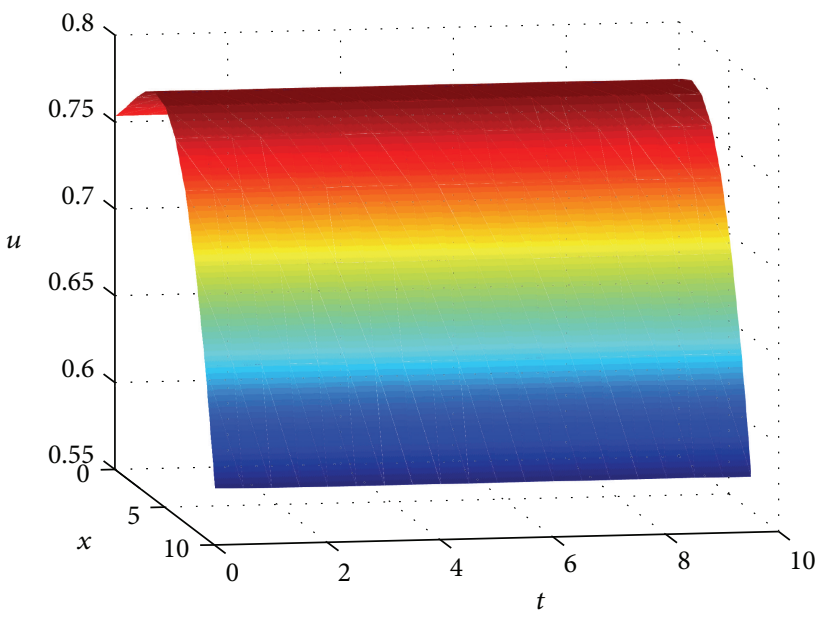

(a)

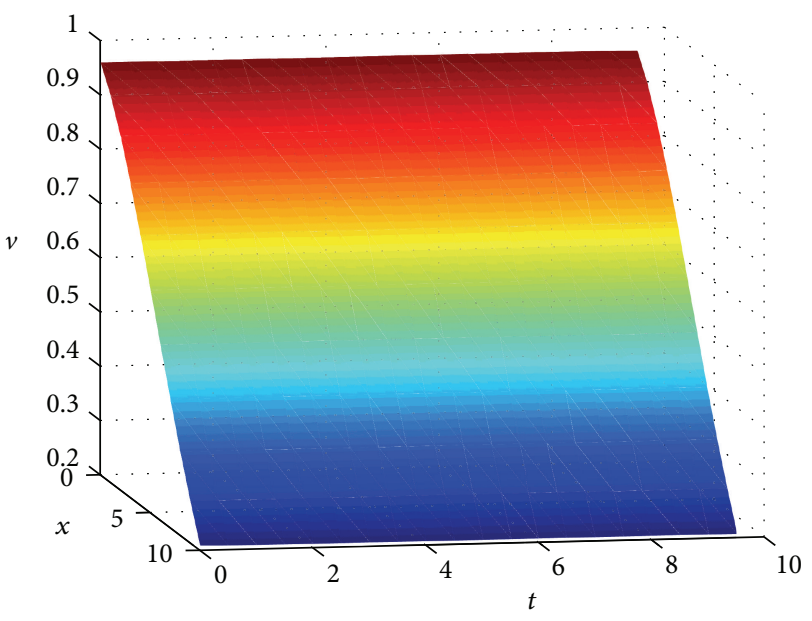

(b)

FIGURE 2: Numerical simulation for (5) with $u_{0}=\cos (x / 10), v_{0}=\cos (x / 10)$.

can be shown in Figure 2, and it indicates that the solution is convergent to a stationary solution.

\section{Conclusion}

In this paper, it can be seen from the numerical results that the global attractor $\mathscr{A}$ of problem (5) exists for certain $\delta$ and $P$. It includes several types of periodic solutions and quasiperiodic solutions. Besides, the solutions also reduce to stationary solution in some parameter value. All these numerical simulations are consistent with our theoretical conclusions.

\section{Conflict of Interests}

The authors declare that there is no conflict of interests.

\section{Acknowledgments}

This work is supported by National Natural Science Foundation of China (11272277, 11471129), Innovation Scientists and Technicians Troop Construction Projects of Henan Province (134100510013), Innovative Research Team in University of Henan Province (13IRTSTHN019), and Key Project of the Education Department Henan Province (15A110043).

\section{References}

[1] R. A. Satnoianu, P. K. Maini, and M. Menzinger, "Parameter space analysis, pattern sensitivity and model comparison for Turing and stationary flow-distributed waves," Physica D. Nonlinear Phenomena, vol. 160, no. 1-2, pp. 79-102, 2001.

[2] Y. Tang and J. Wang, "Bifurcation analysis on a reactor model with combination of quadratic and cubic steps," Journal of Mathematical Chemistry, vol. 46, no. 4, pp. 1394-1408, 2009.

[3] Y. You, "Global dynamics of the Brusselator equations," Dynamics of Partial Differential Equations, vol. 4, no. 2, pp. 167-196, 2007.
[4] Y. You, "Global dynamics and robustness of reversible autocatalytic reaction-diffusion systems," Nonlinear Analysis. Theory, Methods \& Applications, vol. 75, no. 6, pp. 3049-3071, 2012.

[5] J. Wei and M. Winter, "Existence and stability of multiple-spot solutions for the Gray-Scott model in $R^{2}$," Physica D. Nonlinear Phenomena, vol. 176, no. 3-4, pp. 147-180, 2003.

[6] Y. You, "Asymptotical dynamics of the modified Schnackenberg equations," Discrete and Continuous Dynamical Systems, vol. 2009, pp. 857-868, 2009.

[7] J. M. Ball, "Continuity properties and global attractors of generalized semiflows and the Navier-Stokes equations," in Mechanics: From Theory to Computation, pp. 447-474, Springer, New York, NY, USA, 2000.

[8] X. Chen and J. Shen, "Global attractor for a chemotaxis model with reaction term," Journal of Applied Mathematics, vol. 2013, Article ID 536381, 8 pages, 2013.

[9] X.-Y. Chen and J.-W. Shen, "Research of an othmer-stevens chemotax is model with reproduction term," Journal of Applied Sciences, vol. 13, no. 21, pp. 4456-4462, 2013.

[10] Q. Zheng and J. Shen, "Dynamics and pattern formation in a cancer network with diffusion," Communications in Nonlinear Science and Numerical Simulation, vol. 27, no. 1-3, pp. 93-109, 2015.

[11] Q. Zheng and J. Shen, "Pattern formation in the FitzHughNagumo model," Computers \& Mathematics with Applications, vol. 70, no. 5, pp. 1082-1097, 2015.

[12] Y. You and S. Zhou, "Global dissipative dynamics of the extended Brusselator system," Nonlinear Analysis: Real World Applications, vol. 13, no. 6, pp. 2767-2789, 2012.

[13] C. Sun and C. Zhong, "Attractors for the semilinear reactiondiffusion equation with distribution derivatives in unbounded domains," Nonlinear Analysis: Theory, Methods \& Applications, vol. 63, no. 1, pp. 49-65, 2005.

[14] C.-K. Zhong, M.-H. Yang, and C.-Y. Sun, "The existence of global attractors for the norm-to-weak continuous semigroup and application to the nonlinear reaction-diffusion equations," Journal of Differential Equations, vol. 223, no. 2, pp. 367-399, 2006. 


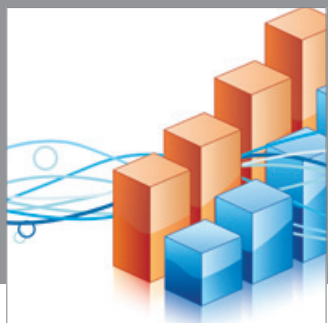

Advances in

Operations Research

mansans

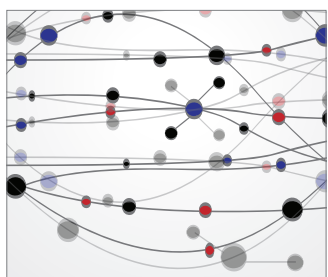

The Scientific World Journal
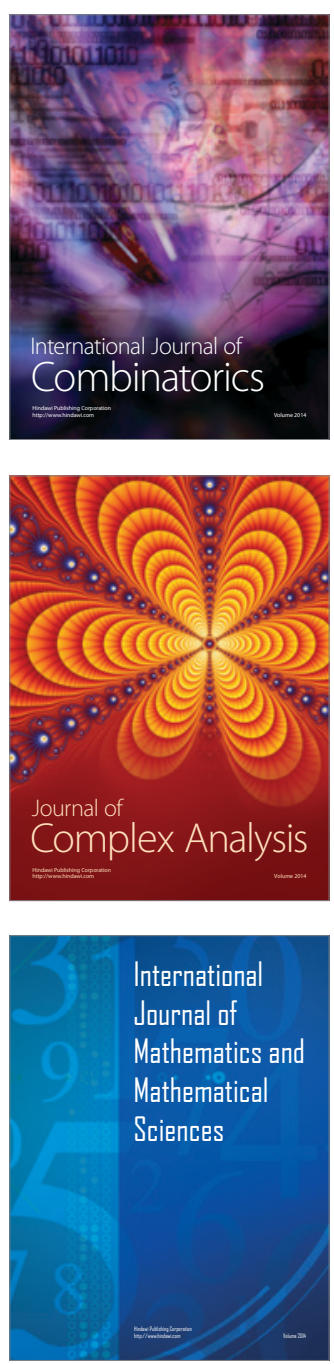
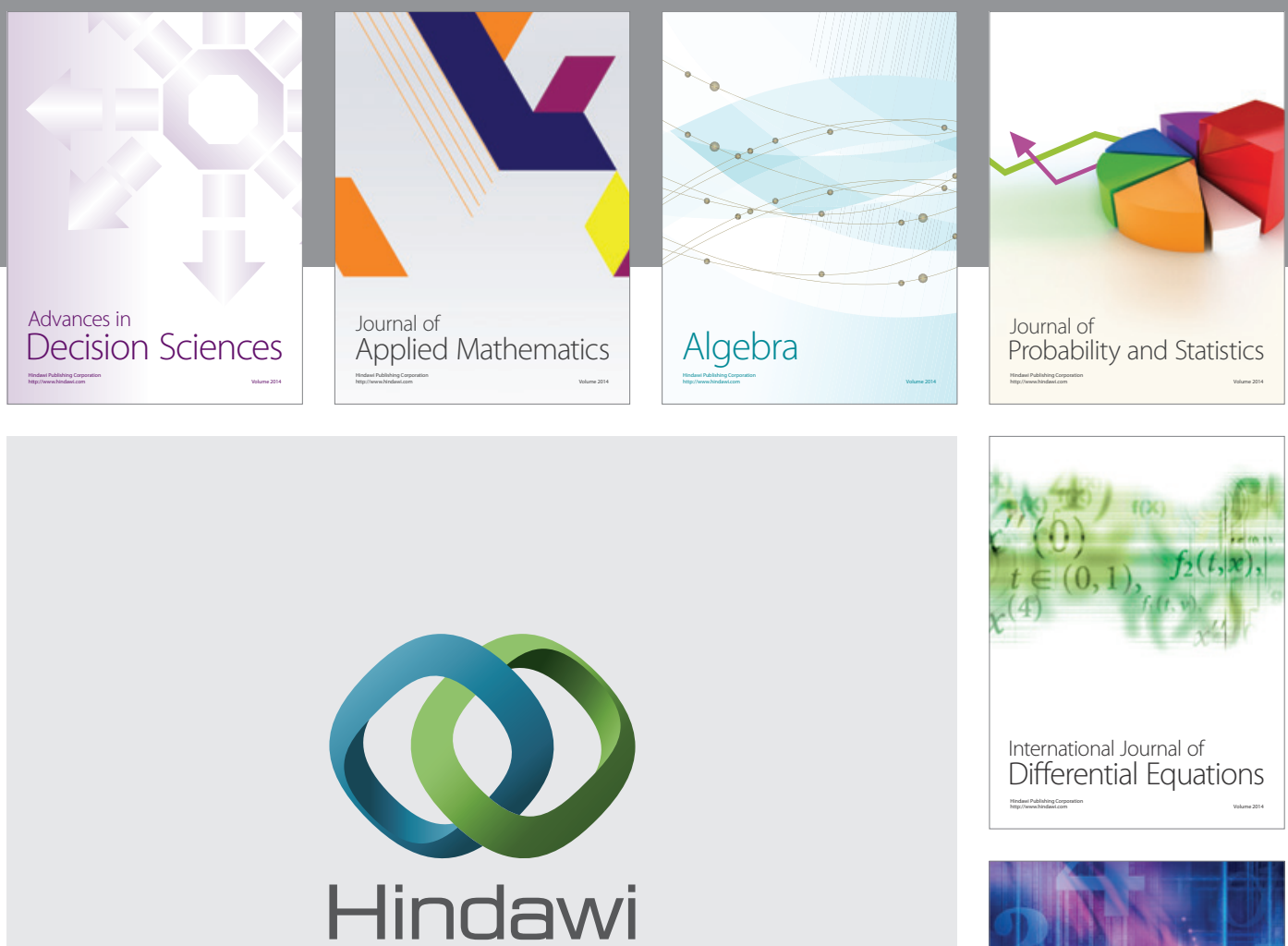

Submit your manuscripts at http://www.hindawi.com
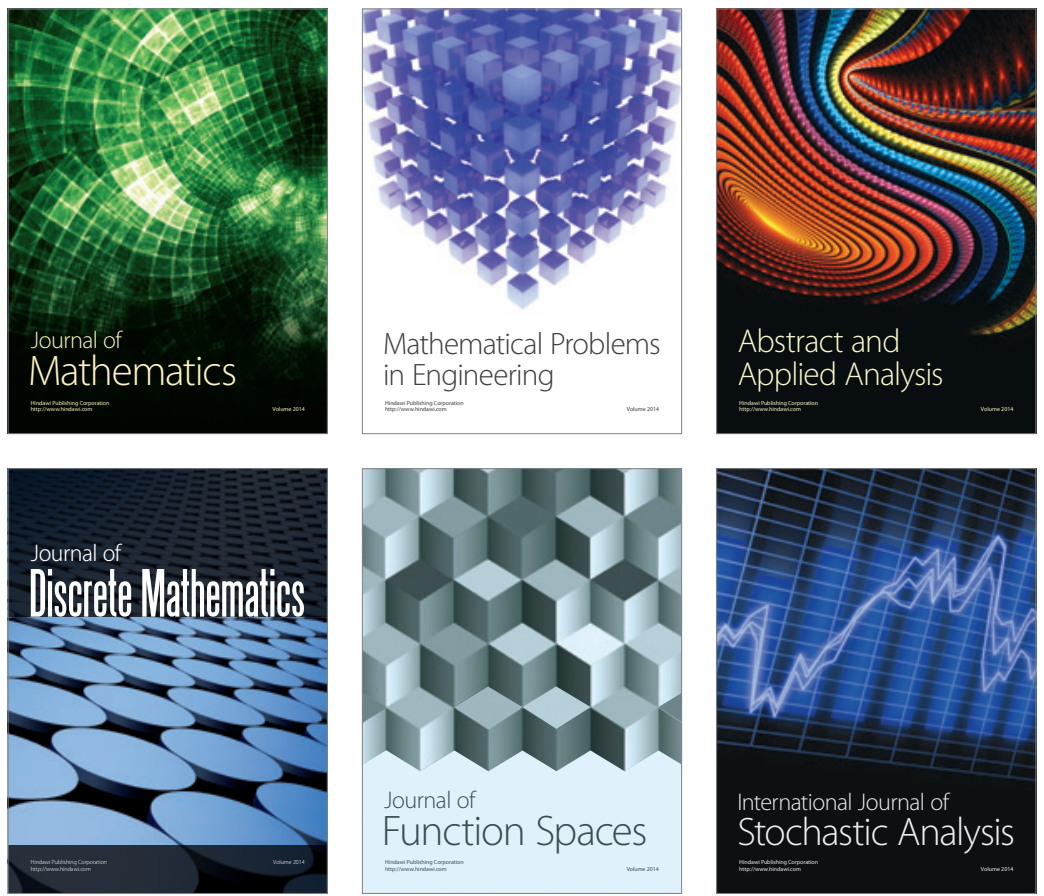

Journal of

Function Spaces

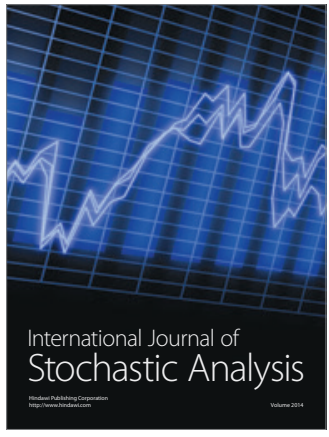

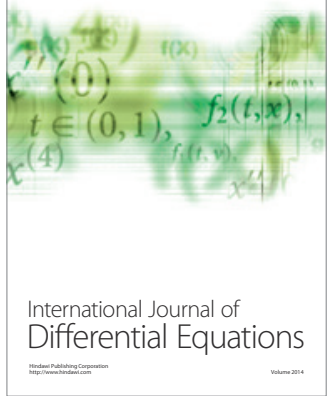
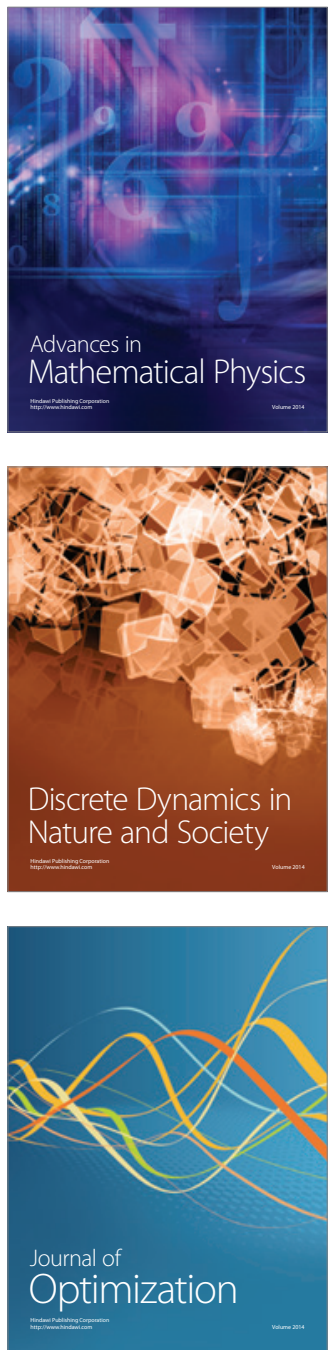